\title{
Is Breast Background Parenchymal Enhancement on MRI Related to BI-RADS Score and Follow-Up Rate?
}

\author{
Afsaneh Alikhassi', Hedieh Akbari' ${ }^{1}$, Seyedeh Nooshin Miratashi Yazdi', Sona Akbari Kia1, \\ Farzin Roozafzai \\ ${ }^{1}$ Department of Radiology, Cancer Institute, Imam Khomeini Hospital, Tehran University of Medical Sciences, Tehran, Iran \\ ${ }^{2}$ Research Institute, Shariati Hospital, Tehran University of Medical Sciences, Tehran, Iran \\ Email:a-alikhasi@sina.tums.ac.ir, afsanehalikhassi@yahoo.co.uk
}

How to cite this paper: Alikhassi, A., Akbari, H., Yazdi, S.N.M., Kia, S.A. and Roozafzai, F. (2018) Is Breast Background Parenchymal Enhancement on MRI Related to BI-RADS Score and Follow-Up Rate? Advances in Breast Cancer Research, 7, 15-22.

https://doi.org/10.4236/abcr.2018.71002

Received: December 15, 2017

Accepted: January 7, 2018

Published: January 10, 2018

Copyright $\odot 2018$ by authors and Scientific Research Publishing Inc. This work is licensed under the Creative Commons Attribution International License (CC BY 4.0).

http://creativecommons.org/licenses/by/4.0/

c) (i) Open Access

\begin{abstract}
Objective: We investigated the correlations between background parenchymal enhancement (BPE) and MRI interpretations with respect to short-interval follow-ups and biopsy rates. Methods: All accessible MRI examinations from 128 women during a limited time period in 2016 were evaluated. A blinded radiologist visually categorized $\mathrm{BPE}$ as minimal, mild, moderate, or marked. A BI-RADS category was also assigned. We used descriptive statistics to report the findings and chi-square and Fisher's exact tests to compare categories. Results: Prevalence of minimal, mild, moderate, and marked BPE was $14.1 \%$, $43.0 \%, 32.0 \%$, and $10.9 \%$, respectively. The short-interval follow-up rates were $22.2 \%, 27.3 \%, 26.8 \%$, and $7.1 \%$ in women with minimal, mild, moderate, and marked BPE, respectively. BPE was not associated with the short-interval follow-up rate $(\mathrm{p}$-value $=0.477$ ). Biopsy rates were $22.2 \%, 27.3 \%, 22.0 \%$, and $57.1 \%$ in women with minimal, mild, moderate, and marked BPE, respectively. Although there was no significant relationship between biopsy rates and BPE levels ( $p$-value $=0.095$ ) in the total population, these two factors were significantly associated in premenopausal women ( $\mathrm{p}$-value $=0.023$ ) and in women of $30-39$ years ( $\mathrm{p}$-value $=0.001)$. Conclusion: Higher BPE does not correlate with short-interval follow-up rates, but appears to be related to biopsy rate, thus causing false-positives and unnecessary biopsy recommendations, particularly in younger, premenopausal women.
\end{abstract}

\section{Keywords}

Breast MRI, Background Parenchymal Enhancement, BI-RADS, Short-Interval Follow-Up, Biopsy 


\section{Introduction}

Background parenchymal enhancement (BPE), representing normal fibro-glandular tissue enhancement in dynamic contrast-enhanced magnetic resonance imaging (MRI), corresponds to hormonally responsive glandular tissue [1]. BPE may represent blood flow and hormonal activity of dense tissue [2]. Histamine-like effects of estrogen cause vasodilation and increase breast tissue vascular permeability, and the proliferative effects of progesterone increase metabolic activity resulting in increased perfusion of breast [3] [4]. BPE is higher in younger women with hormonally active breasts [5] [6]. BPE varies between patients and is currently reported using the American College of Radiology Breast Imaging Reporting and Data System (BI-RADS) [7].

MRI is the most sensitive imaging method for detection of breast cancer (BC), yet it has limited specificity [8] [9]. Some authors have suggested that an increased BPE has the possibility to obscure breast lesions and decrease MRI sensitivity for detection of $\mathrm{BC}$ or may even be misinterpreted as a suspicious finding itself, thus leading to an increased false-positive biopsy rate and short-interval follow-up rate [4] [10] [11] [12], but some others believe BPE does not significantly affect the diagnostic accuracy of breast MRIs [13]. Imaging material and hormonal status also affect BPE and can limit breast MRI interpretation [14] [15]. We aimed to investigate the correlations between BPE and the interpretation of MRI examinations with respect to BI-RADS scores, short-interval follow-up rates, and biopsy rates.

\section{Materials and Methods}

Design: From January 2016 to January 2017 and after taking written informed consents, we randomly included 128 women who had breast MRI in a tertiary referral hospital to investigate the correlation between BPE and BI-RADS score. All MRI images retrospectively were observed by a specialized breast radiologist. Indications for breast MRI were questionable mammographic or ultra-sonographic findings, unexplainable clinical findings, BIRADS 3 lesions' follow-ups, and planning for 12 new cases of biopsy-proven malignancies. The exclusion criteria consisted of a history of conservative breast surgery, chemotherapy, radiotherapy, and/or hormone replacement therapy.

In a dedicated surface breast coil, the same techniques for all patients were performed in a 1.5 Tesla MRI scanner, and a blinded expert breast radiologist reviewed all of the images. Localization, T1-weighted non-fat-suppressed sequences, and $\mathrm{T} 2$-weighted fat-suppressed sequences were conducted following standard protocols, and six sequences after injection of $0.1 \mathrm{mmol} / \mathrm{L}$ gadopentetate dimeglumine (Magnevist, Bayer and Germany) were then obtained and subtracted pixel-by-pixel from the first non-contrast images. The radiologist visually assessed BPE in post-contrast fat-suppressed T1-weighted subtracted images and categorized BPE on the basis of fifth edition of BI-RADS criteria as minimal, mild, moderate, or marked [7]. 
BI-RADS categories consisted of 6 groups: 0 (incomplete assessment, recall); 1 (normal findings, routine screening); 2 (benign findings, routine screening); 3 (probably benign findings, short-interval follow-up); 4 (suspicious findings, biopsy); 5 (highly-suspicious findings, biopsy); and 6 (biopsy-proven malignancy, excision). Biopsies were taken with a Tru-cut needle from patients who had been recommended for a biopsy based on BI-RADS 4 or 5 which was given by another radiologist who was blind to the study. Following standard protocols, an expert pathologist examined breast specimens' biopsies from patients who had been classified as BI-RADS 4 or 5 (Figures 1-3).

Data analysis: We used descriptive statistics (count, frequency distributions) to report BI-RADS categories, short-interval follow-up and biopsy rates, and positive predictive biopsy value (PPV). Comparing categories, we used chi-square and Fisher's exact tests. Type I error was considered 0.05. The data was collected on MS Office Excel datasheets (Microsoft, Redmond, USA). All analyses were conducted using SPSS v.22 (IBM Corp., Armonk, USA).

Ethical considerations: The study protocol was evaluated and approved by the Atieh hospital institutional ethics committee considering the retrospective setting of the study and taking written informed consents from patients for using their data.

Confidentiality of information was followed. Researchers caused no adverse or harmful events to patients. Authors were committed to the principles of the Declaration of Helsinki and declared no conflicts of interests.

\section{Results}

We studied 89 (69.5\%) pre-menopausal and 39 (30.5\%) postmenopausal women aged 18 to 74 years old. Approximately a third of the women (count $=44$ ) were aged 30 - 39 years. Minimal, mild, moderate, and marked BPE were observed in $18(14.1 \%), 55(43.0 \%), 41$ (32.0\%), and 14 (10.9\%) women, respectively.

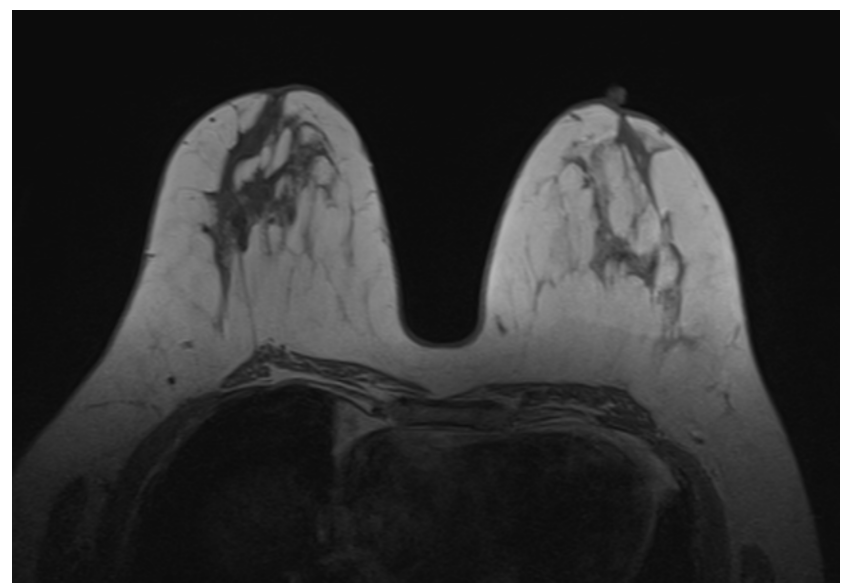

(a)

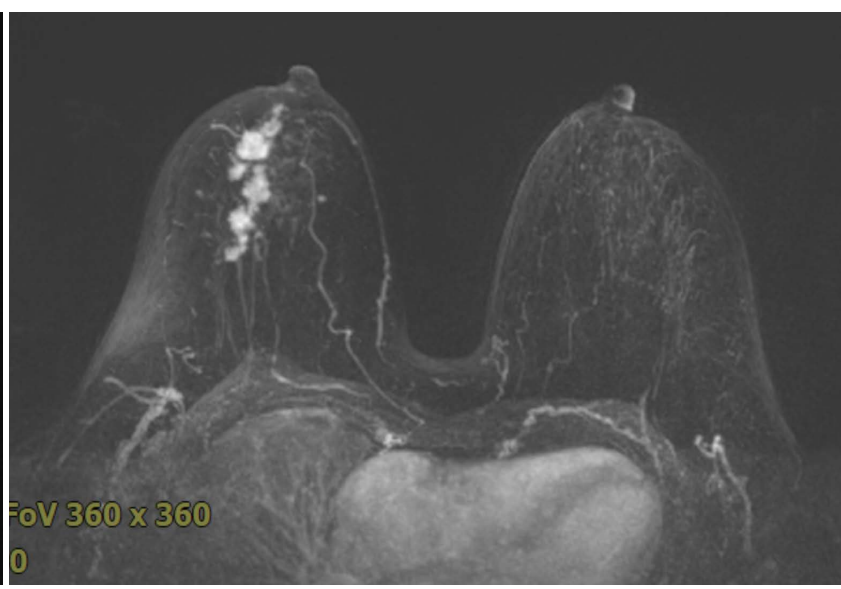

(b)

Figure 1. Breast MRI in a 40 years old woman with right breast mass sensation which was proved to be a cancer regarding biopsy under ultrasound guide. In T1 sequence, scattered fibroglandular tissue is seen (a). After contrast injection in a mild background parenchymal enhancement, multiple irregular masses with segmental distribution are seen in right breast lateral part (b). 


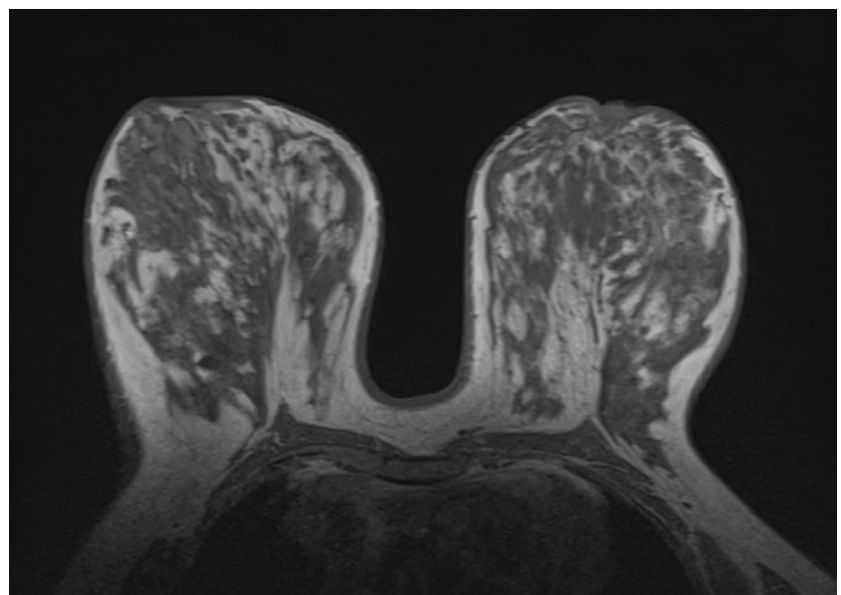

(a)

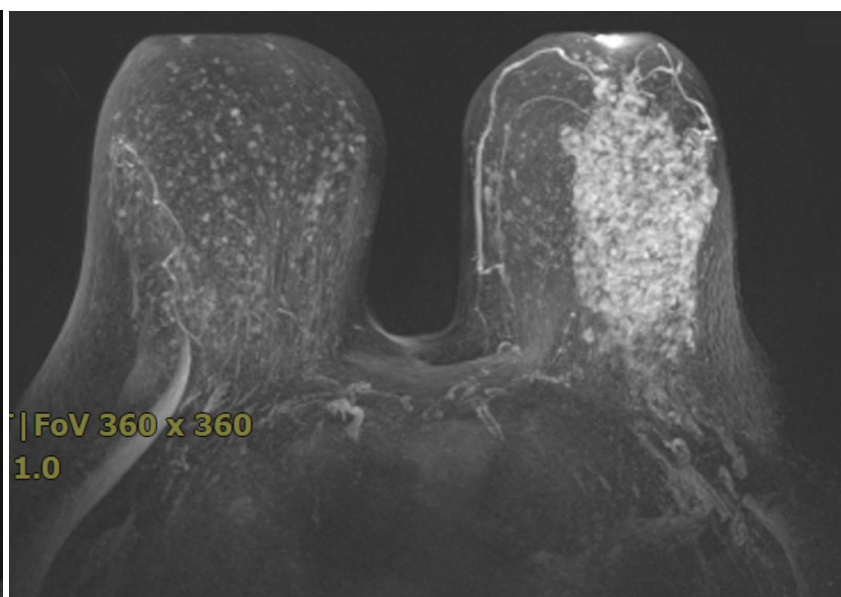

(b)

Figure 2. This is MRI of a 45 years old woman with an extremely dense mammography. In T1 sequence before contrast, extremely compact fibroglandular tissue is seen in breasts (a). Maximum intensity projection images (MIP) shows a large area with non-mass like clumped nodular enhancement in a moderate nodular background parenchymal enhancement context which was proved to be extensive ductal carcinoma in situ with some foci of invasive ductal carcinoma (b).

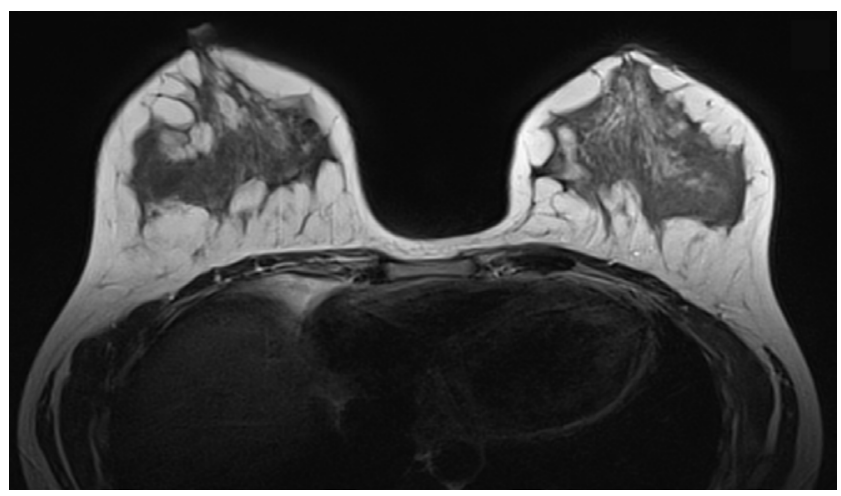

(a)

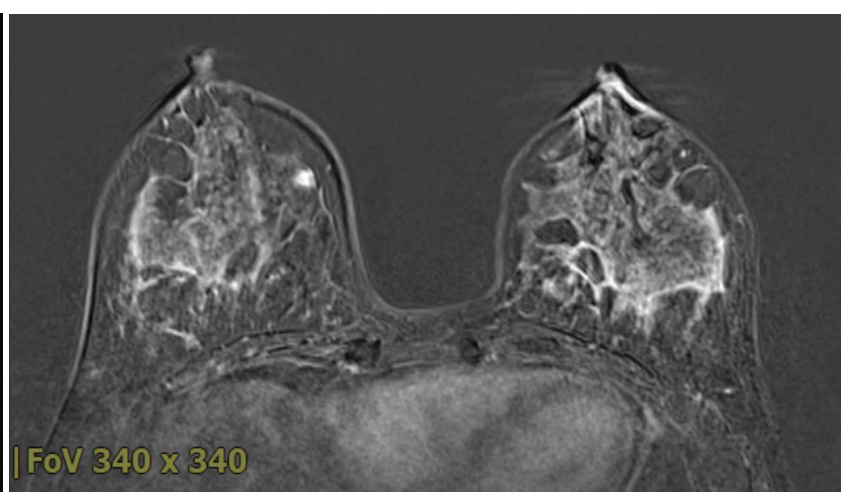

(b)

Figure 3. Breast MRI in a middle age woman with heterogenous type of fibroglandular tissue in T1 sequences without contrast (a) and moderate background parenchymal enhancement after contrast (b), a small mildly irregular shape early enhancing mass is seen in right breast central inner part which was given BIRADS 4 and then after with benign pathology's result in biopsy.

BPE and BI-RADS score level, Table 1 represents a significant association between BI-RADS score and BPE level (Fisher's exact p-value $=0.023$ ). According to a sub-analysis, the association remained significant in premenopausal women (Fisher's exact p-value $=0.031$ ) and in women 30 - 39 years (Fisher's exact $\mathrm{p}$-value $=0.024)$.

BPE and short term follow up, overall, 49 women $(38.3 \%)$ presented with normal or benign examinations (such as BI-RADS 1 or 2) and continued routine screening. About $55 \%$ of women with minimal BPE were assigned a BI-RADS category of 1 or 2 versus $36.4 \%, 39.0 \%$, and $21.4 \%$ of women with mild, moderate, and marked BPE, respectively (Table 2).

As shown in Table 2, 31 women (24.2\%) were assigned to BI-RADS category 3 (probably benign) and underwent short-interval follow-ups. The short-interval follow-up rate was $22.2 \%, 27.3 \%, 26.8 \%$, and $7.1 \%$ in women with minimal, 
Table 1. Cross-tabulation of "background parenchymal enhancement" level and BI-RADS score in breast magnetic resonance imaging of 128 women. No one was assigned to a BI-RADS category of 0 (incomplete assessment).

\begin{tabular}{lccccc}
\hline & \multicolumn{5}{c}{ Background Parenchymal Enhancement } \\
\hline \multicolumn{1}{c}{ BI-RADS category } & Total $^{\mathrm{a}}$ & $\begin{array}{c}\text { Minimal } \\
(\mathrm{n}=18)\end{array}$ & $\begin{array}{c}\text { Mild } \\
(\mathrm{n}=55)\end{array}$ & $\begin{array}{c}\text { Moderate } \\
(\mathrm{n}=41)\end{array}$ & $\begin{array}{c}\text { Marked } \\
(\mathrm{n}=14)\end{array}$ \\
\hline 1) Normal findings & $5(3.9)$ & $3(16.7)$ & $1(1.8)$ & $0(0.0)$ & $1(7.1)$ \\
2) Benign findings & $44(34.4)$ & $7(38.9)$ & $19(34.5)$ & $16(39.0)$ & $2(14.3)$ \\
3) Probably benign findings & $31(24.2)$ & $4(22.2)$ & $15(27.3)$ & $11(26.8)$ & $1(7.1)$ \\
4) Suspicious findings & $32(25.0)$ & $2(11.1)$ & $13(23.6)$ & $9(22.0)$ & $8(57.1)$ \\
5) Highly-suspicious findings & $4(3.1)$ & $2(11.1)$ & $2(3.6)$ & $0(0.0)$ & $0(0.0)$ \\
6) Biopsy-proven malignancy & $12(9.4)$ & $0(0.0)$ & $5(9.1)$ & $5(12.2)$ & $2(14.3)$ \\
\hline
\end{tabular}

BI-RADS: breast imaging-reporting and data system, n: count. ${ }^{a}$ numbers represent "count (percentage within each column)".

Table 2. Associations of routine screening rate, short-interval follow-up rate, biopsy rate, and positive predictive value of biopsy with "background parenchymal enhancement" level in breast magnetic resonance imaging of 128 women.

\begin{tabular}{cccccccc}
\hline & \multicolumn{5}{c}{ Background Parenchymal Enhancement $^{\mathrm{e}}$} & \\
\hline MRI interpretation & Total $^{\mathrm{e}}$ & $\begin{array}{c}\text { Minimal, } \\
\mathrm{n}=18\end{array}$ & $\begin{array}{c}\text { Mild, } \\
\mathrm{n}=55\end{array}$ & $\begin{array}{c}\text { Moderate, } \\
\mathrm{n}=41\end{array}$ & $\begin{array}{c}\text { Marked, } \\
\mathrm{n}=14\end{array}$ & p-value* $^{*}$ \\
\hline $\begin{array}{c}\text { Recommended routine } \\
\text { screening }^{\mathrm{a}}\end{array}$ & $38.3(49)$ & $55.6(10)$ & $36.4(20)$ & $39.0(16)$ & $21.4(3)$ & 0.274 \\
$\begin{array}{c}\text { Recommended } \\
\text { short-interval follow-up }\end{array}$ & $24.2(31)$ & $22.2(4)$ & $27.3(15)$ & $26.8(11)$ & $7.1(1)$ & 0.477 \\
$\begin{array}{c}\text { Recommended biopsy } \\
\text { ( }\end{array}$ & $28.1(36)$ & $22.2(4)$ & $27.3(15)$ & $22.0(9)$ & $57.1(8)$ & 0.095 \\
PPV for biopsy $^{\text {d }}$ & $16.7(6)$ & $50.0(2)$ & $20.0(3)$ & $0.0(0)$ & $12.5(1)$ & 0.107 \\
\hline
\end{tabular}

n: count, PPV: positive predictive value; ${ }^{\mathrm{a} I-R A D S} 1$ or 2 ; ${ }^{\mathrm{b}} \mathrm{BI}-\mathrm{RADS} 3$; ${ }^{\mathrm{c}} \mathrm{BI}-\mathrm{RADS} 4$ or 5 ; ${ }^{\mathrm{d}}$ percentage of malignant lesions detected in women who underwent biopsy; ${ }^{\mathrm{e}}$ numbers represent "percentage within each column (count)"; *Fisher's exact test.

mild, moderate, and marked BPE, respectively. BPE was not associated with short-interval follow-up rates ( $\mathrm{p}$-value $=0.477$ ).

BPE and biopsy, biopsies were recommended for 36 women (28.1\%) with BI-RADS category of 4 or 5. Table 2 shows the rate and PPV (positive predictive value) of biopsy according to BPE levels. The biopsy rates were $22.2 \%, 27.3 \%$, $22.0 \%$, and $57.1 \%$ for women with minimal, mild, moderate, and marked BPE, respectively. Although there were no significant relationships between the biopsy rates and BPE levels ( $\mathrm{p}$-value $=0.095)$ in the total population, a sub-analysis showed significant associations between BPE and biopsy rates in premenopausal women (Fisher's exact p-value $=0.023$ ) and in women $30-39$ years (Fisher's exact $\mathrm{p}$-value $=0.001$ ).

Biopsies proved the presence of malignancies (invasive ductal carcinoma) in six patients, giving a PPV of $16.7 \%$. PPV for biopsy did not associate with BPE in the total population $(\mathrm{p}$-value $=0.107)$. 
According to sub-analyses, PPV for biopsy was $16.7 \%$ in both premenopausal $(4 / 24)$ and postmenopausal (2/12) women and 8.3\% (1/12) in women aged 30 39 years. PPV for biopsy and age decade were not associated (Fisher's exact $\mathrm{p}$-value $=0.318$.

\section{Discussion}

Few studies have evaluated the impact of BPE on MRI diagnostic performance. In our study, BPE associated with BI-RADS scores in all women, including premenopausal ones and women aged 30 - 39 years. De Martini et al. reported that abnormal interpretation rates for women with moderate or marked BPE were significantly higher than that for women with minimal or mild BPE, but positive biopsy rates were not affected by different BPE [13]. However, in a study by Hambly in women with minimal BPE, no significant difference in BI-RADS category for women with mild, moderate, or marked BPE was found [4].

In contrast to the De Martini et al. study [13], we considered the relationships between BPE and the menopausal status variable.

Almost quarter of our subjects underwent short-interval follow-ups and rates of short-interval follow-ups did not associate with BPE; these results are in contrast to the previous Hambly study [4]. They showed an association of short-interval follow-ups with BPE levels but the rate of BIRADS 3 in their study was greater than usual (43.6\%).

In agreement with Hambly et al. [4], biopsy rates did not correlate with BPE in our study. Yet, it was associated with BPE in sub-groups of premenopausal women and in women living their third decade although the PPV for biopsy was limited.

Only one out of six biopsies revealed a malignancy in our study. In agreement with Hambly et al. and De Martini et al. [4] [13], our study showed that PPV was not affected by BPE regardless of menopausal status or age decade.

According to low predictive values of biopsy in premenopausal women and women aged 30 - 39 years in our study, and the point that BPE is higher in younger breasts [16] [17], it can be implied that increased BPE, misinterpreted as malignancy, may lead to false-positive diagnosis.

Our study like Hambly and colleagues [4] included only patients who underwent biopsy, so sensitivity and specificity were not measured.

There were some limitations to this study as: small sample size and visual qualitative BPE assessment. In addition, being a single-centered study is a challenge for making generalizations about the results. One other limitation was assigning a single BI-RADS assessment to both breasts because the breast with the lower BI-RADS category data was lost. It is especially important when women with BI-RADS category 4 or 5 in one breast had BI-RADS category 3 in the contralateral breast.

\section{Conclusion}

Higher BPE on breast MRIs does not correlate with short-interval follow-up 
rates but can increase false-positive interpretations, leading to unnecessary biopsies, particularly in younger, premenopausal women.

\section{Authors' Contributions}

AA designed the concept. All authors collaborated in data acquisition. FR provided statistical advice on study design and data analyses. AA and FR analyzed and interpreted the data. SAK drafted the manuscript. All authors critically reviewed the manuscript. All authors read and approved the final manuscript. AA takes responsibility for the paper as a whole.

\section{Conflict of Interest}

The authors declare that there is no conflict of interest.

\section{Source of Funding}

This research received no specific grant from any funding agency in the public, commercial.

\section{References}

[1] Bennani-Baiti, B., Dietzel, M. and Baltzer, P.A. (2016) MRI Background Parenchymal Enhancement Is Not Associated with Breast Cancer. PLoS ONE, 11, 7. https://doi.org/10.1371/journal.pone.0158573

[2] Pike, M.C. and Pearce, C.L. (2013) Mammographic Density, MRI Background Parenchymal Enhancement and Breast Cancer Risk. Annals of Oncology: Official Journal of the European Society for Medical Oncology, 24, 37-41.

[3] Zeppa, R. (1969) Vascular Response of the Breast to Estrogen. The Journal of Clinical Endocrinology and Metabolism, 29, 695-700. https://doi.org/10.1210/jcem-29-5-695

[4] Hambly, N.M., Liberman, L., Dershaw, D.D., Brennan, S. and Morris, E.A. (2011) Background Parenchymal Enhancement on Baseline Screening Breast MRI: Impact on Biopsy Rate and Short-Interval Follow-Up. AJR American Journal of Roentgenology, 196, 218-224. https://doi.org/10.2214/AJR.10.4550

[5] King, V., Brooks, J.D., Bernstein, J.L., Reiner, A.S., Pike, M.C. and Morris, E.A. (2011) Background Parenchymal Enhancement at Breast MR Imaging and Breast Cancer Risk. Radiology, 260, 50-60. https://doi.org/10.1148/radiol.11102156

[6] Muller-Schimpfle, M., Ohmenhauser, K., Stoll, P., Dietz, K. and Claussen, C.D. (1997) Menstrual Cycle and Age: Influence on Parenchymal Contrast Medium Enhancement in MR Imaging of the Breast. Radiology, 203, 145-149.

https://doi.org/10.1148/radiology.203.1.9122383

[7] Morris, E.A., Comstock, C.E. and Lee, C.H. (2013) ACR BI-RADS ${ }^{\circledast}$ Magnetic Resonance Imaging. In: ACR BI-RADS Atlas, Breast Imaging Reporting and Data System, Reston, VA, American College of Radiology.

[8] Menezes, G.L., Knuttel, F.M., Stehouwer, B.L., Pijnappel, R.M. and Van den Bosch, M.A. (2014) Magnetic Resonance Imaging in Breast Cancer: A Literature Review and Future Perspectives. World Journal of Clinical Oncology, 5, 61-70. https://doi.org/10.5306/wjco.v5.i2.61

[9] Kuhl, C.K. (2007) Current Status of Breast MR Imaging. Part 2. Clinical Applica- 
tions. Radiology, 244, 672-691. https://doi.org/10.1148/radiol.2443051661

[10] Orel, S.G. and Schnall, M.D. (2001) MR Imaging of the Breast for the Detection, Diagnosis, and Staging of Breast Cancer. Radiology, 220, 13-30. https://doi.org/10.1148/radiology.220.1.r01j13113

[11] Kawamura, A., Satake, H., Ishigaki, S., Ikeda, M., Kimura, R., Shimamoto, K. and Shinji, N. (2015) Prediction of Background Parenchymal Enhancement on Breast MRI Using Mammography, Ultrasonography, and Diffusion-Weighted Imaging. Nagoya Journal of Medical Science, 77, 425-437.

[12] Kuhl, C. (2007) The Current Status of Breast MR Imaging. Part I. Choice of Technique, Image Interpretation, Diagnostic Accuracy, and Transfer to Clinical Practice. Radiology, 244, 356-378. https://doi.org/10.1148/radiol.2442051620

[13] De Martini, W.B., Liu, F., Peacock, S., Eby, P.R., Gutierrez, R.L. and Lehman, C.D. (2012) Background Parenchymal Enhancement on Breast MRI: Impact on Diagnostic Performance. AJR American Journal of Roentgenology, 198, 373-380. https://doi.org/10.2214/AJR.10.6272

[14] Telegrafo, M., Rella, L., Stabile Ianora, A.A., Angelelli, G. and Moschetta, M. (2016) Breast MRI Background Parenchymal Enhancement (BPE) Correlates with the Risk of Breast Cancer. Magnetic Resonance Imaging, 34, 173-176. https://doi.org/10.1016/j.mri.2015.10.014

[15] Telegrafo, M., Rella, L., Stabile Ianora, A.A., Angelelli, G. and Moschetta, M. (2016) Effect of Background Parenchymal Enhancement on Breast Cancer Detection with Magnetic Resonance Imaging. Diagnostic and Interventional Imaging, 97, 315-320. https://doi.org/10.1016/j.diii.2015.12.006

[16] Giess, C.S., Yeh, E.D., Raza, S. and Birdwell, R.L. (2014) Background Parenchymal Enhancement at Breast MR Imaging: Normal Patterns, Diagnostic Challenges, and Potential for False-Positive and False-Negative Interpretation. Radiographics: $A$ Review Publication of the Radiological Society of North America, 34, 234-247. https://doi.org/10.1148/rg.341135034

[17] Vogel, P.M., Georgiade, N.G., Fetter, B.F., Vogel, F.S. and McCarty, K.S. (1981) The Correlation of Histologic Changes in the Human Breast with the Menstrual Cycle. The American Journal of Pathology, 104, 23-34. 